Sādhanā Vol. 36, Part 3, June 2011, pp. 411-423. (c) Indian Academy of Sciences

\title{
A two-dimensional problem for a fibre-reinforced anisotropic thermoelastic half-space with energy dissipation
}

\author{
IBRAHIM A ABBAS \\ Department of mathematics, Faculty of Science, Sohag University, Sohag, \\ 82524, Egypt \\ Department of mathematics, Faculty of Science, Jazan University, Jazan, \\ 82822, Saudi Arabia \\ e-mail: ibrabbas7@yahoo.com
}

MS received 5 May 2010; revised 3 November 2010; accepted 4 March 2011

\begin{abstract}
The theory of thermoelasticity with energy dissipation is employed to study plane waves in a fibre-reinforced anisotropic thermoelastic half-space. We apply a thermal shock on the surface of the half-space which is taken to be traction free. The problem is solved numerically using a finite element method. Moreover, the numerical solutions of the non-dimensional governing partial differential equations of the problem are shown graphically. Comparisons are made with the results predicted by Green-Naghdi theory of the two types (GNII without energy dissipation) and (GNIII with energy dissipation). We found that the reinforcement has great effect on the distribution of field quantities. Results carried out in this paper can be used to design various fibre-reinforced anisotropic thermoelastic elements under thermal load to meet special engineering requirements.
\end{abstract}

Keywords. Fibre-reinforced; finite element method; thermoelasticity with energy dissipation.

\section{Introduction}

Fibre-reinforced composites are used in a variety of structures due to their low weight and high strength. The mechanical behaviour of many fibre-reinforced composite materials is adequately modelled by the theory of linear elasticity for transversely isotropic materials, with the preferred direction coinciding with the fibre direction. In such composites the fibres are usually arranged in parallel straight lines. The characteristic property of a reinforced composite is that its components act together as a single anisotropic unit as long as they remain in the elastic condition. Sengupta \& Nath (2001) discussed the problem of surface waves in fibre-reinforced anisotropic elastic media. Singh (2001) showed that, for wave propagation in fibre-reinforced anisotropic media, this decoupling cannot be achieved by the introduction of the displacement potentials. Hashin \& Rosen (1964) gave the elastic moduli for fibre-reinforced materials. In classical dynamical coupled theory of thermoelasticity, the thermal and mechanical waves propagate with an infinite velocity, which is not physically admissible. 
The theory of couple thermoelasticity was extended by Lord \& Shulman (1967) and Green \& Lindsay (1972) by including the thermal relaxation time in constitutive relations. The theory was extended for anisotropic body by Dhaliwal \& Sherief (1980). Green \& Naghdi (1993) proposed a new generalized thermoelasticity theory by including the thermal-displacement gradient among the independent constitutive variables. An important feature of this theory, which is not present in other thermoelasticity theories, is that it does not accommodate dissipation of thermal energy. The relevant fundamental aspects of this theory are contained in Grenn \& Naghdi (1991; 1992). Singh (2006) studied the wave propagation in thermally conducting linear fibre-reinforced composite materials with one relaxation time.

The exact solution of the governing equations of the generalized thermoelasticity theory for a coupled and nonlinear/linear system exists only for very special and simple initial and boundary problems. A numerical solution technique is used to calculate the solution of general problems. For this reason the finite element method is chosen.

The Finite element method is a powerful technique originally developed for numerical solution of complex problems in structural mechanics, and it remains the method of choice for complex systems. A further benefit of this method is that it allows physical effects to be visualized and quantified regardless of experimental limitations. On the other hand, the finite element method in different generalized thermoelastic problems has been applied by many authors (see for instance Tian et al 2006; Abbas 2007; Abbas \& Abd-alla 2008; Abbas \& Othman 2009; Youssef \& Abbas 2007).

In the present work, the Green and Naghdi theory is applied to study the influence of reinforcement on the total deformation body and the interaction with each other. Furthermore, the problem is solved numerically using a finite element method (FEM). Numerical results for the temperature distribution, displacement and the stress components are represented graphically. As an application of the results carried out in this paper, we can design various fibre-reinforced anisotropic thermoelastic elements under thermal load to meet special engineering requirements.

\section{Basic equations and formulation}

According to Green \& Nagdhi (1993) and Singh (2006), the linear equations governing thermoelastic interactions in homogeneous anisotropic solid in the absence of body force and heat sources are as follows:

$$
\begin{aligned}
\tau_{i j, j} & =\rho \ddot{u}_{i}, \quad i, j=1,2,3, \\
K^{*} T_{, i j}+K_{i j} \dot{T}_{, i j} & =\rho c_{e} \ddot{T}+T_{o} \beta_{i j} \ddot{u}_{i, j}, \quad i, j=1,2,3 .
\end{aligned}
$$

The constitutive equation for a fibre-reinforced linearly thermoelastic anisotropic medium whose preferred direction is that of a unit vector a will be as follows (Belfield et al (1983):

$$
\begin{aligned}
\tau_{\mathrm{ij}}= & \lambda \mathrm{e}_{\mathrm{kk}} \delta_{\mathrm{ij}}+2 \mu_{\mathrm{T}} \mathrm{e}_{\mathrm{ij}}+\alpha\left(\mathrm{a}_{\mathrm{k}} \mathrm{a}_{\mathrm{m}} \mathrm{e}_{\mathrm{km}} \delta_{\mathrm{ij}}+\mathrm{a}_{\mathrm{i}} \mathrm{a}_{\mathrm{j}} \mathrm{e}_{\mathrm{kk}}\right) \\
& +2\left(\mu_{\mathrm{L}}-\mu_{\mathrm{T}}\right)\left(\mathrm{a}_{\mathrm{i}} \mathrm{a}_{\mathrm{k}} \mathrm{e}_{\mathrm{kj}}+\mathrm{a}_{\mathrm{j}} \mathrm{a}_{\mathrm{k}} \mathrm{e}_{\mathrm{ki}}\right) \\
& +\beta \mathrm{a}_{\mathrm{k}} \mathrm{a}_{\mathrm{m}} \mathrm{e}_{\mathrm{km}} \mathrm{a}_{\mathrm{i}} \mathrm{a}_{\mathrm{j}}-\beta_{\mathrm{ij}}\left(\mathrm{T}-\mathrm{T}_{0}\right) \delta_{\mathrm{ij}}, \quad i, j, k, m=1,2,3,
\end{aligned}
$$

where $\rho$ is the mass density; $u_{i}$ the displacement vectors components; $e_{i j}$ the strain tensor; $\tau_{i j}$ the stress tensor; $T$ the temperature change of a material particle; $T_{o}$ the reference uniform temperature of the body; $\beta_{i j}$ the thermal elastic coupling tensor; $c_{e}$ the specific heat at constant strain; $K_{i j}$ the thermal conductivity; $K^{*}$ the material characteristic of the theory; $\lambda, \mu_{T}$ are elastic parameters; $\alpha, \beta,\left(\mu_{L}-\mu_{T}\right)$ are reinforced anisotropic elastic parameters and $\mathbf{a} \equiv\left(\mathrm{a}_{1}, \mathrm{a}_{2}, \mathrm{a}_{3}\right)$, 
$a_{1}^{2}+a_{2}^{2}+a_{3}^{2}=1$. The comma notation is used for spatial derivatives and superimposed dot represents time differentiation.

We consider the problem of a fibre-reinforced anisotropic half-space $\left(x_{1} \geq 0\right)$ All the considered functions will depend on the time $t$ and the coordinates $x_{1}$ and $x_{2}$ Thus, the displacement vector $u_{i}$ will have the components

$$
u=u_{1}=u\left(x_{1}, x_{2}, t\right), v=u_{2}=v\left(x_{1}, x_{2}, t\right), w=u_{3}=0 .
$$

We choose the fibre-direction as $\mathbf{a} \equiv(1,0,0)$ so that the preferred direction is the $x_{1}$ axis and Eqs. (1), (2) and (3) are simplified to be

$$
\begin{gathered}
\tau_{11}=\left(\lambda+2 \alpha+4 \mu_{\mathrm{L}}-2 \mu_{T}+\beta\right) \frac{\partial \mathrm{u}}{\partial \mathrm{x}_{1}}+(\lambda+\alpha) \frac{\partial \mathrm{v}}{\partial \mathrm{x}_{2}}-\beta_{11}\left(\mathrm{~T}-\mathrm{T}_{\mathrm{o}}\right), \\
\tau_{22}=\left(\lambda+2 \mu_{T}\right) \frac{\partial v}{\partial \mathrm{x}_{2}}+(\lambda+\alpha) \frac{\partial u}{\partial \mathrm{x}_{1}}-\beta_{22}\left(\mathrm{~T}-\mathrm{T}_{\mathrm{o}}\right) \\
\tau_{12}=\mu_{\mathrm{L}}\left(\frac{\partial v}{\partial \mathrm{x}_{1}}+\frac{\partial u}{\partial \mathrm{x}_{2}}\right), \\
\mathrm{A}_{11} \frac{\partial^{2} \mathrm{u}}{\partial x_{1}^{2}}+\mathrm{A}_{12} \frac{\partial^{2} \mathrm{v}}{\partial x_{1} \partial x_{2}}+A_{13} \frac{\partial^{2} \mathrm{u}}{\partial x_{2}^{2}}-\beta_{11} \frac{\partial T}{\partial x_{1}}=\rho \frac{\partial^{2} \mathrm{u}}{\partial \mathrm{t}^{2}} \\
\mathrm{~A}_{22} \frac{\partial^{2} v}{\partial x_{2}^{2}}+\mathrm{A}_{12} \frac{\partial^{2} u}{\partial x_{1} \partial x_{2}}+A_{13} \frac{\partial^{2} v}{\partial x_{1}^{2}}-\beta_{22} \frac{\partial \mathrm{T}}{\partial x_{2}}=\rho \frac{\partial^{2} v}{\partial \mathrm{t}^{2}} \\
\left(\mathrm{~K}^{*}+\mathrm{K}_{11} \frac{\partial}{\partial t}\right) \frac{\partial^{2} T}{\partial x_{1}^{2}}+\left(\mathrm{K}^{*}+\mathrm{K}_{22} \frac{\partial}{\partial t}\right) \frac{\partial^{2} T}{\partial x_{2}^{2}}=\rho c_{e} \frac{\partial^{2} T}{\partial t^{2}}+T_{o} \frac{\partial^{2}}{\partial t^{2}}\left(\beta_{11} \frac{\partial u}{\partial x_{1}}+\beta_{22} \frac{\partial v}{\partial x_{2}}\right),
\end{gathered}
$$

with

$$
\begin{gathered}
A_{11}=\lambda+2\left(\alpha+\mu_{T}\right)+4\left(\mu_{\mathrm{L}}-\mu_{T}\right)+\beta, A_{12}=\alpha+\lambda, A_{13}=\mu_{\mathrm{L}}, A_{22}=\lambda+2 \mu_{T}, \\
\beta_{11}=\left(2 \lambda+3 \alpha+4 \mu_{L}-2 \mu_{T}+\beta\right) \alpha_{11}+(\lambda+\alpha) \alpha_{22}, \beta_{22}=(2 \lambda+\alpha) \alpha_{11}+\left(\lambda+2 \mu_{T}\right) \alpha_{22},
\end{gathered}
$$
where $\alpha_{11}, \alpha_{22}$ are coefficients of linear thermal expansion. For convenience, the following nondimensional variables are used:

$$
\begin{aligned}
& \left(x^{\prime}, y^{\prime}, u^{\prime}, v^{\prime}\right)=c \chi(x, y, u, v), t^{\prime}=c^{2} \chi t, \quad T^{\prime}=\frac{\beta_{11}\left(T-T_{0}\right)}{\rho c^{2}}, \\
& \left(\tau_{11}^{\prime}, \tau_{22}^{\prime}, \tau_{12}^{\prime}\right)=\frac{1}{\rho c^{2}}\left(\tau_{11}, \tau_{22}, \tau_{12}\right), c^{2}=\frac{A_{11}}{\rho}, \chi=\frac{\rho c_{e}}{K_{11}} .
\end{aligned}
$$

In terms of the non-dimensional quantities defined in Eqs. (11), the above governing equations reduce to (dropping the dashed for convenience)

$$
\begin{gathered}
\tau_{11}=\frac{\partial \mathrm{u}}{\partial \mathrm{x}_{1}}+B_{1} \frac{\partial \mathrm{v}}{\partial \mathrm{x}_{2}}-\mathrm{T}, \\
\tau_{22}=B_{1} \frac{\partial u}{\partial \mathrm{x}_{1}}+B_{2} \frac{\partial v}{\partial \mathrm{x}_{2}}-B_{3} \mathrm{~T},
\end{gathered}
$$




$$
\begin{gathered}
\tau_{12}=B_{4}\left(\frac{\partial v}{\partial \mathrm{x}_{1}}+\frac{\partial u}{\partial \mathrm{x}_{2}}\right), \\
\frac{\partial^{2} \mathrm{u}}{\partial x_{1}^{2}}+\left(B_{1}+B_{4}\right) \frac{\partial^{2} \mathrm{v}}{\partial x_{1} \partial x_{2}}+B_{4} \frac{\partial^{2} \mathrm{u}}{\partial x_{2}^{2}}-\frac{\partial \mathrm{T}}{\partial x_{1}}=\frac{\partial^{2} \mathrm{u}}{\partial \mathrm{t}^{2}} \\
B_{2} \frac{\partial^{2} v}{\partial x_{2}^{2}}+\left(B_{1}+B_{4}\right) \frac{\partial^{2} u}{\partial x_{1} \partial x_{2}}+B_{4} \frac{\partial^{2} v}{\partial x_{1}^{2}}-B_{3} \frac{\partial T}{\partial x_{2}}=\frac{\partial^{2} v}{\partial t^{2}}, \\
\left(\varepsilon_{2}+\varepsilon_{3} \frac{\partial}{\partial t}\right) \frac{\partial^{2} T}{\partial x_{1}^{2}}+\left(\varepsilon_{2}+\varepsilon_{3} \frac{K_{22}}{K_{11}} \frac{\partial}{\partial t}\right) \frac{\partial^{2} T}{\partial x_{2}^{2}}=\frac{\partial^{2} T}{\partial t^{2}}+\frac{\partial^{2}}{\partial t^{2}}\left(\varepsilon_{0} \frac{\partial u}{\partial x_{1}}+\varepsilon_{1} \frac{\partial v}{\partial x_{2}}\right),
\end{gathered}
$$

where

$$
\begin{aligned}
& \left(B_{1}, B_{2}, B_{4}\right)=\frac{1}{A_{11}}\left(A_{12}, A_{22}, A_{13}\right), B_{3}=\frac{\beta_{22}}{\beta_{11}},\left(\varepsilon_{0}, \varepsilon_{1}\right)=\frac{T_{o} \beta_{11}}{A_{11} \rho c_{e}}\left(\beta_{11}, \beta_{22}\right), \\
& \left(\varepsilon_{2}, \varepsilon_{3}\right)=\frac{1}{\rho c_{e}}\left(\frac{K^{*}}{c^{2}}, K_{11} \chi\right) .
\end{aligned}
$$

\section{Finite element formulation}

In this section, the governing equations of generalized thermoelasticity are summarized, followed by the corresponding finite element equations. In the finite element method, the eight-node isoparametric, quadrilateral element is used for displacement components and temperature calculations. The displacement component $\{u\}$ and the temperature $T$ are related to the corresponding nodal values $\left\{u^{e}\right\}$ and $\left\{T^{e}\right\}$ by

$$
\{u\}=[N]\left\{u^{e}\right\},\{T\}=\left[N^{\prime}\right]\left\{T^{e}\right\},
$$

where $[N]$ and $\left[N^{\prime}\right]$ are shape functions which are given by

$$
[N]=\left[\begin{array}{ccccccc}
N_{1} & 0 & N_{2} & 0 & \ldots & N_{n} & 0 \\
0 & N_{1} & 0 & N_{2} & \ldots & 0 & N_{n}
\end{array}\right], \quad\left[\begin{array}{l}
N^{\prime}
\end{array}\right]=\left\{\begin{array}{cccc}
N_{1} & N_{2} & \ldots & N_{n}
\end{array}\right\},
$$

where $\mathrm{n}$ denotes the number of nodes in the grid. According to the geometrical equation as the following:

$$
e_{i j}=\frac{1}{2}\left(u_{i, j}+u_{j, i}\right)
$$

As well as $T^{\prime}=T_{, i}$, it yields

$$
\{e\}=\left[D_{1}\right]\left\{u^{e}\right\}, T^{\prime}=\left[D_{2}\right]\left\{T^{e}\right\} .
$$

The variational form of the above equation is

$$
\delta\{e\}=\left[D_{1}\right] \delta\left\{u^{e}\right\}, \delta T^{\prime}=\left[D_{2}\right] \delta\left\{T^{e}\right\} .
$$


If the coordinates $x_{1}$ and $x_{2}$ are considered then $\left[D_{1}\right]$ and $\left[D_{2}\right]$ have the following forms, respectively:

$$
\begin{gathered}
{\left[D_{1}\right]=\left[\begin{array}{ccccccc}
\frac{\partial N_{1}}{\partial x_{1}} & 0 & \frac{\partial N_{2}}{\partial x_{1}} & 0 & \ldots & \frac{\partial N_{n}}{\partial x_{1}} & 0 \\
0 & \frac{\partial N_{1}}{\partial x_{2}} & 0 & \frac{\partial N_{2}}{\partial x_{2}} & \ldots & 0 & \frac{\partial N_{n}}{\partial x_{2}} \\
\frac{\partial N_{1}}{\partial x_{2}} & \frac{\partial N_{1}}{\partial x_{1}} & \frac{\partial N_{2}}{\partial x_{2}} & \frac{\partial N_{2}}{\partial x_{1}} & \ldots & \frac{\partial N_{n}}{\partial x_{2}} & \frac{\partial N_{n}}{\partial x_{1}}
\end{array}\right]} \\
{\left[D_{2}\right]=\left[\begin{array}{ccccc}
\frac{\partial N_{1}}{\partial x_{1}} & \frac{\partial N_{2}}{\partial x_{1}} & \ldots & \frac{\partial N_{n}}{\partial x_{1}} \\
\frac{\partial N_{1}}{\partial x_{2}} & \frac{\partial N_{2}}{\partial x_{2}} & \ldots & \frac{\partial N_{n}}{\partial x_{2}}
\end{array}\right]}
\end{gathered}
$$

The virtual displacement principle of domain $V$ and its boundary $A$ can be written as

$$
\begin{aligned}
& \int_{V}\left(\delta\{e\}^{T}\left\{\tau_{i j}\right\}+\delta\left\{T^{\prime}\right\}\left[K^{*} T_{, i}+K_{i i} \dot{T}_{, i}\right]\right) d V \\
& \quad+\int_{V}\left(\delta\left\{u^{e}\right\} \rho \ddot{u}_{i}+\delta\left\{T^{e}\right\}\left[\rho c_{e} \ddot{T}+T_{o} \beta_{i j} \ddot{u}_{i, j}\right]\right) d V=\int_{A}\left(\delta\left\{u^{e}\right\}^{T}\{\bar{\tau}\}+\delta\left\{T^{e}\right\} \bar{T}\right) d A,
\end{aligned}
$$

where $\{\bar{\tau}\}$ represents the components of the traction vector, and $\bar{T}$ represents the heat flux. In equation (25), the governing equations are multiplied by weighting functions and then are integrated over the spatial domain $V$ with the boundary $A$. Applying integration by parts and making use of the divergence theorem reduce the order of the spatial derivatives and allows for the application of the boundary conditions. Thus, the finite element equations corresponding to equations (15), (16) and (17) can be obtained as

$$
\begin{gathered}
\sum_{e=1}^{n}\left(\left[\begin{array}{ccc}
M_{11}^{e} & 0 & 0 \\
0 & M_{22}^{e} & 0 \\
M_{31}^{e} & M_{32}^{e} & M_{33}^{e}
\end{array}\right]\left\{\begin{array}{c}
\ddot{u}^{e} \\
\ddot{v}^{e} \\
\ddot{T}^{e}
\end{array}\right\}+\left[\begin{array}{ccc}
0 & 0 & 0 \\
0 & 0 & 0 \\
0 & 0 & C_{33}^{e}
\end{array}\right]\left\{\begin{array}{c}
\dot{u}^{e} \\
\dot{v}^{e} \\
\dot{T}^{e}
\end{array}\right\}\right. \\
\left.+\left[\begin{array}{ccc}
K_{11}^{e} & K_{12}^{e} & K_{13}^{e} \\
K_{21}^{e} & K_{22}^{e} & K_{23}^{e} \\
0 & 0 & K_{33}^{e}
\end{array}\right]\left\{\begin{array}{c}
u^{e} \\
v^{e} \\
T^{e}
\end{array}\right\}=\left\{\begin{array}{c}
F_{1}^{e} \\
F_{2}^{e} \\
F_{3}^{e}
\end{array}\right\}\right),
\end{gathered}
$$

where the coefficients in equation (26) are given below:

$$
\begin{aligned}
M_{11}^{e} & =\int_{V}[N]^{T}[N] d V, & M_{22}^{e} & =\int_{V}[N]^{T}[N] d V, \\
M_{31}^{e} & =\int_{V} \varepsilon_{0}[N]^{T}\left[\frac{\partial N}{\partial x_{1}}\right] d V, & M_{32}^{e} & =\int_{V} \varepsilon_{1}[N]^{T}\left[\frac{\partial N}{\partial x_{2}}\right] d V, \\
M_{33}^{e} & =\int_{V}[N]^{T}[N] d V, & C_{33}^{e} & =\int_{V} \varepsilon_{3}[N]^{T}\left[\frac{\partial N}{\partial x_{1}}+\frac{K_{22}}{K_{11}} \frac{\partial N}{\partial x_{2}}\right] d V,
\end{aligned}
$$




$$
\begin{array}{ll}
K_{11}^{e}=\int_{V}\left(\left[\frac{\partial N}{\partial x_{1}}\right]^{T}\left[\frac{\partial N}{\partial x_{1}}\right]+B_{4}\left[\frac{\partial N}{\partial x_{2}}\right]^{T}\left[\frac{\partial N}{\partial x_{2}}\right]\right) d V, & K_{13}^{e}=\int_{V}-\left[\frac{\partial N}{\partial x_{1}}\right]^{T}[N] d V, \\
K_{12}^{e}=\int_{V}\left(B_{1}+B_{4}\right)\left[\frac{\partial N}{\partial x_{2}}\right]^{T}\left[\frac{\partial N}{\partial x_{1}}\right] d V, & K_{23}^{e}=\int_{V}-B_{3}\left[\frac{\partial N}{\partial x_{2}}\right]^{T}[N] d V, \\
K_{21}^{e}=\int_{V}\left(B_{1}+B_{4}\right)\left[\frac{\partial N}{\partial x_{1}}\right]^{T}\left[\frac{\partial N}{\partial x_{2}}\right] d V, & F_{1}^{e}=\int_{A}[N]^{T} \bar{\tau}_{x} d A, \\
K_{22}^{e}=\int_{V}\left(B_{2}\left[\frac{\partial N}{\partial x_{2}}\right]^{T}\left[\frac{\partial N}{\partial x_{2}}\right]+B_{4}\left[\frac{\partial N}{\partial x_{1}}\right]^{T}\left[\frac{\partial N}{\partial x_{1}}\right]\right) d V, & F_{2}^{e}=\int_{A}[N]^{T} \bar{\tau}_{y} d A, \\
K_{33}^{e}=\int_{V} \varepsilon_{2}\left(\left[\frac{\partial N}{\partial x_{1}}\right]^{T}\left[\frac{\partial N}{\partial x_{1}}\right]+\left[\frac{\partial N}{\partial x_{2}}\right]^{T}\left[\frac{\partial N}{\partial x_{2}}\right]\right) d V, & F_{3}^{e}=\int_{A}[N]^{T} \bar{q} d A .
\end{array}
$$

Symbolically, the discretized equations of equations (26) can be written as

$$
M \ddot{d}+C \dot{d}+K d=F^{e x t},
$$

where M, C, K and $F$ represent the mass, damping, stiffness matrices and external force vectors, respectively and $d=\left[\begin{array}{lll}u & v & T\end{array}\right]^{t}$. On the other hand, the time derivatives of the unknown variables have to be determined by Newmark time integration method (see Zienkiewicz \& Taylor 2000; Reddy 1993; Cook et al 1989).

\section{Application}

We consider the problem of a half-space $\Psi$, which is defined as follows:

$$
\Psi=\left\{\left(x_{1}, x_{2}, x_{3}\right): 0 \leq x_{1}<\infty,-\infty<x_{2}<\infty,-\infty<x_{3}<\infty\right\} .
$$

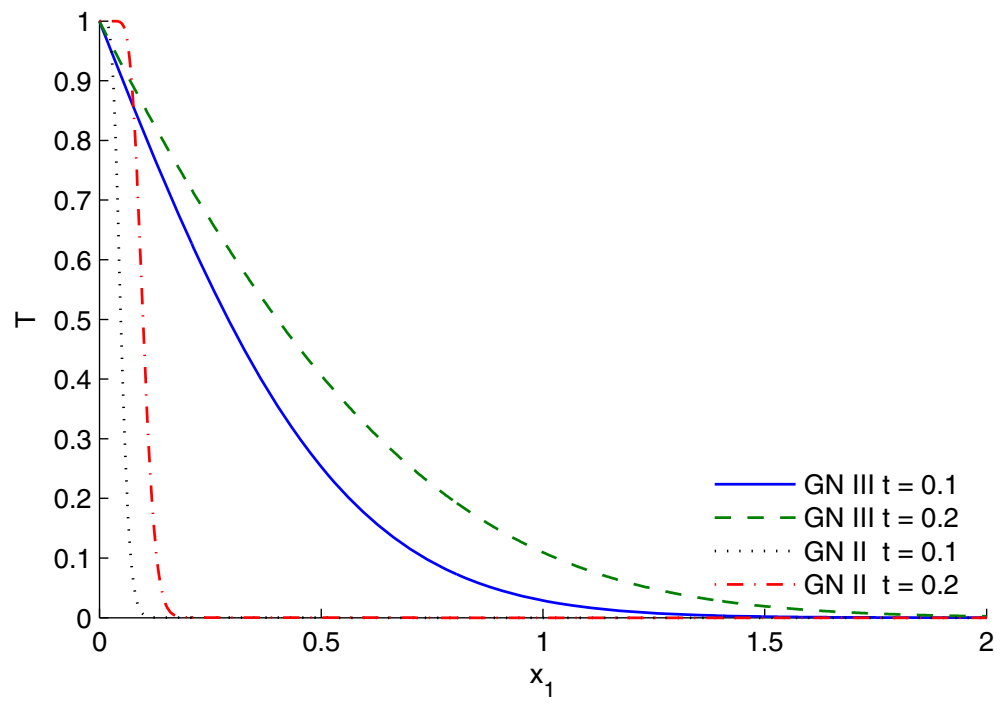

Figure 1. The temperature distribution for different values of $t$ at $x_{2}=1$. 


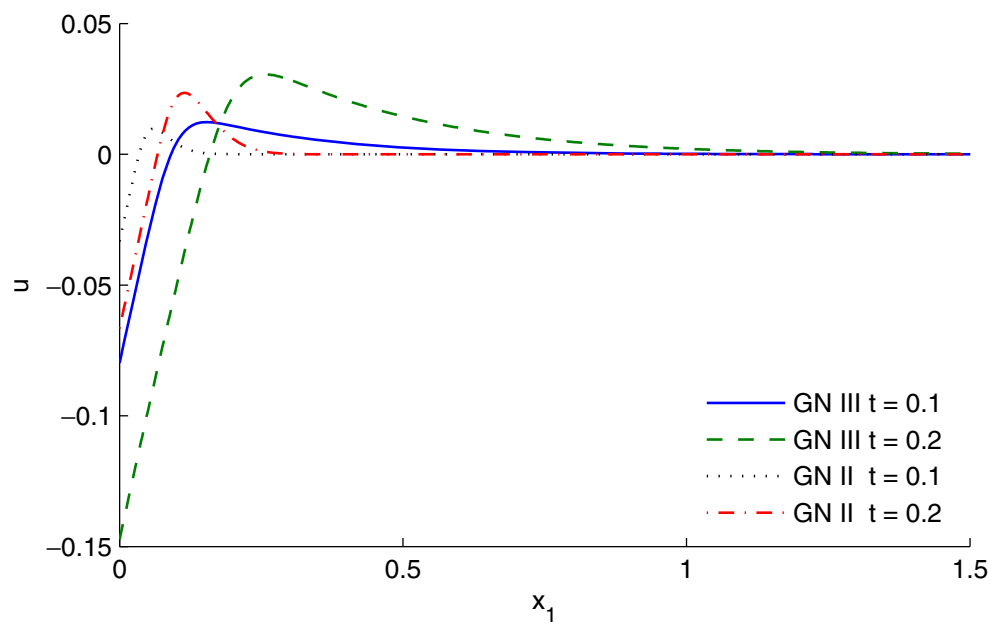

Figure 2. Horizontal displacement distribution $u$ for different values of $t$ at $x_{2}=1$.

The surface of the half-space is taken to be traction free, and the thermal shock $g\left(x_{2}, t\right)$ applied on the surface at $x_{1}=0$ is taken of the form

$$
g\left(x_{2}, t\right)=T_{1} H(t) H\left(l-\left|x_{2}\right|\right),
$$

where $H$ is the Heaviside unit step function and $T_{1}$ is a constant. This means that heat is applied on the surface of the half-space on a narrow band of width $2 l$ surrounding the $x_{2}$-axis to keep it at temperature $T_{1}$, while the rest of the surface is kept at zero temperature. Assuming the following initial conditions:

$$
u=v=T=0 \quad t=0, \quad \dot{u}=\dot{v}=\dot{T}=0 \quad t=0 .
$$

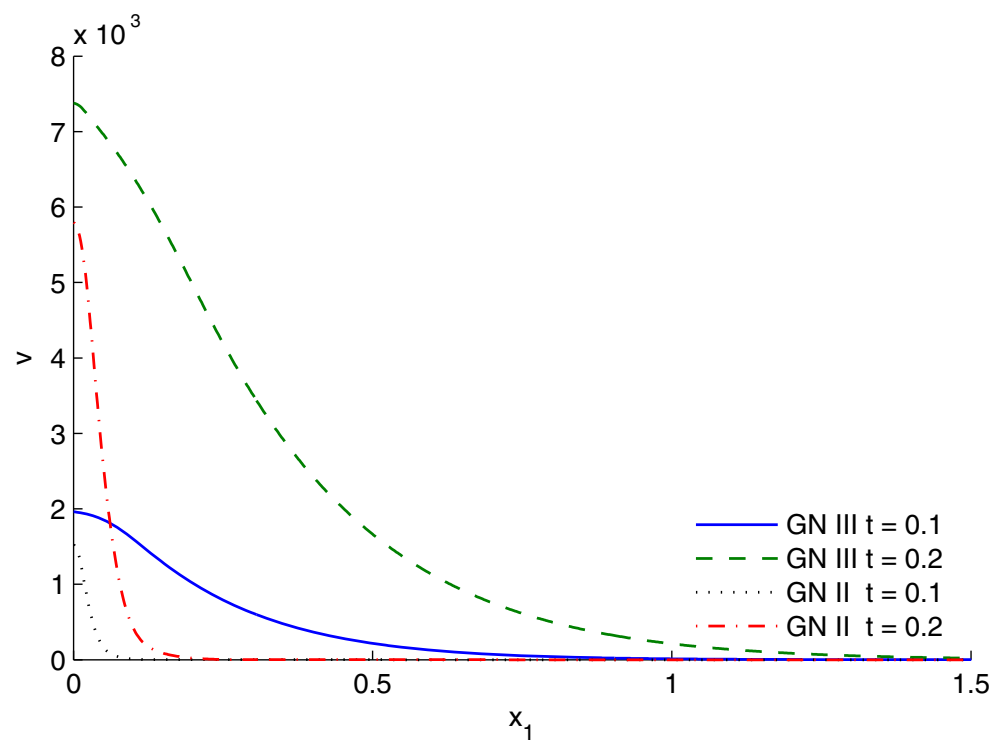

Figure 3. Vertical displacement distribution $v$ for different values of $t$ at $x_{2}=1$. 


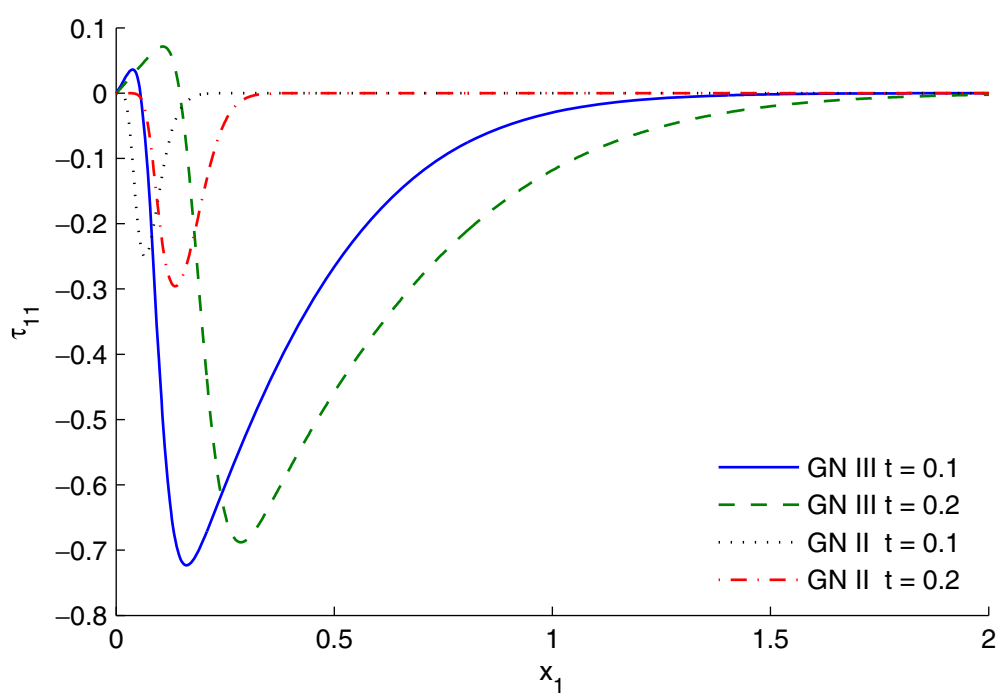

Figure 4. The distribution of stress component $\tau_{11}$ for different values of $t$ at $x_{2}=1$.

\section{Numerical example}

To study the effect of reinforcement on wave propagation, we use the following physical constants for generalized fibre-reinforced thermoelastic materials.

$$
\begin{aligned}
& \rho=2660 \mathrm{~kg} / \mathrm{m}^{3}, \lambda=5.65 \times 10^{10} \mathrm{~N} / \mathrm{m}^{2}, \mu_{\mathrm{T}}=2.46 \times 10^{10} \mathrm{~N} / \mathrm{m}^{2}, \mu_{\mathrm{L}}=5.66 \times 10^{10} \mathrm{~N} / \mathrm{m}^{2}, \\
& \alpha=-1.28 \times 10^{10} \mathrm{~N} / \mathrm{m}^{2}, \beta=220.90 \times 10^{10} \mathrm{~N} / \mathrm{m}^{2}, \alpha_{11}=0.017 \times 10^{-4} \mathrm{deg}^{-1}, \\
& \alpha_{22}=0.015 \times 10^{-4} \mathrm{deg}^{-1}, c_{e}=0.787 \times 10^{3} \mathrm{~J} \mathrm{~kg}^{-1} \mathrm{deg}^{-1}, T_{o}=293 \mathrm{k}, \\
& K_{11}=0.0921 \times 10^{3} \mathrm{~J} \mathrm{~m}^{-1} \mathrm{~s}^{-1} \mathrm{deg}^{-1}, K_{22}=0.0963 \times 10^{3} \mathrm{~J} \mathrm{~m}^{-1} \mathrm{~s}^{-1} \mathrm{deg}^{-1} \\
& T_{1}=1, l=1, \varepsilon_{2}=0.25
\end{aligned}
$$

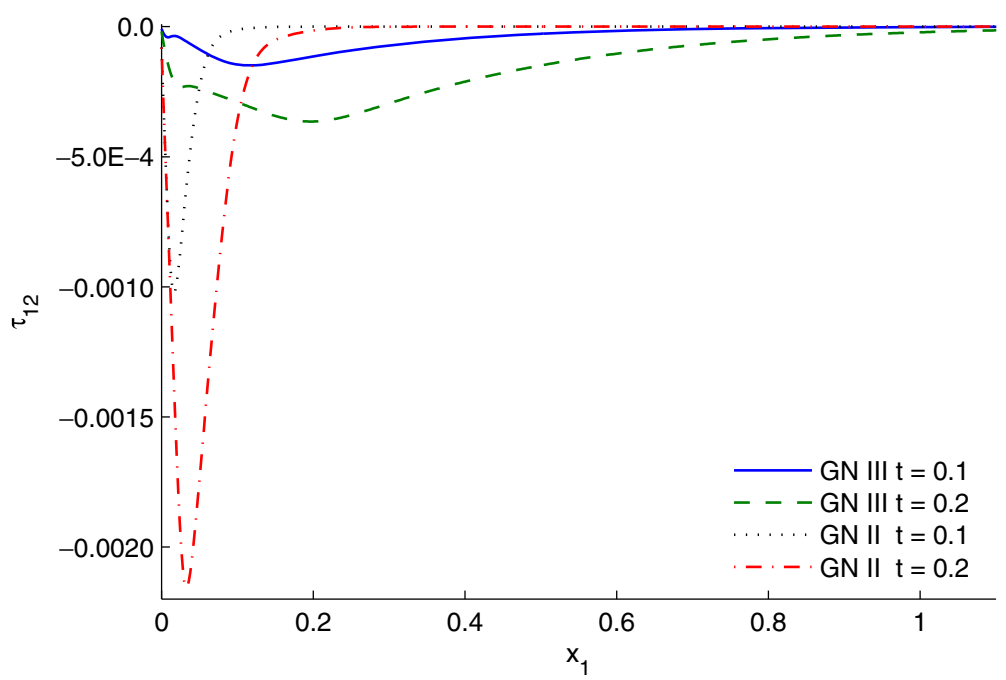

Figure 5. The distribution of stress component $\tau_{12}$ for different values of $t$ at $x_{2}=1$. 


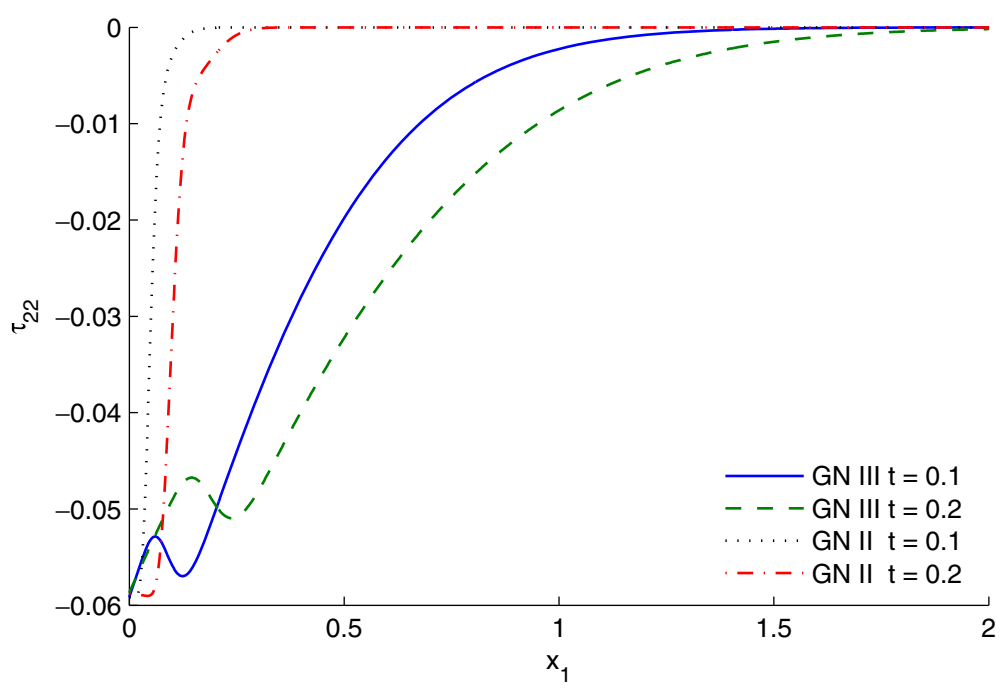

Figure 6. The distribution of stress component $\tau_{22}$ for different values of $t$ at $x_{2}=1$.

Due to the geometrical shape and thermal boundary condition symmetries of the half-space, the problem can be treated as plane strain problem. Only half of the half-space need to be considered because of the symmetries. Calculated results for the response of the non-dimensional temperature $T$, displacement components $u, v$ and stress components $\tau_{11}, \tau_{12}$ and $\tau_{22}$ of the half of the half-space are shown in figures 1-12. These figures represent the solution obtained using the Green-Naghdi theory of type II $\left(\varepsilon_{3}=0\right)$ and type III $\left(\varepsilon_{3}=1\right)$. The Green-Naghdi theory developed a theory of thermoelasticity without energy dissipation whose temperature evolution

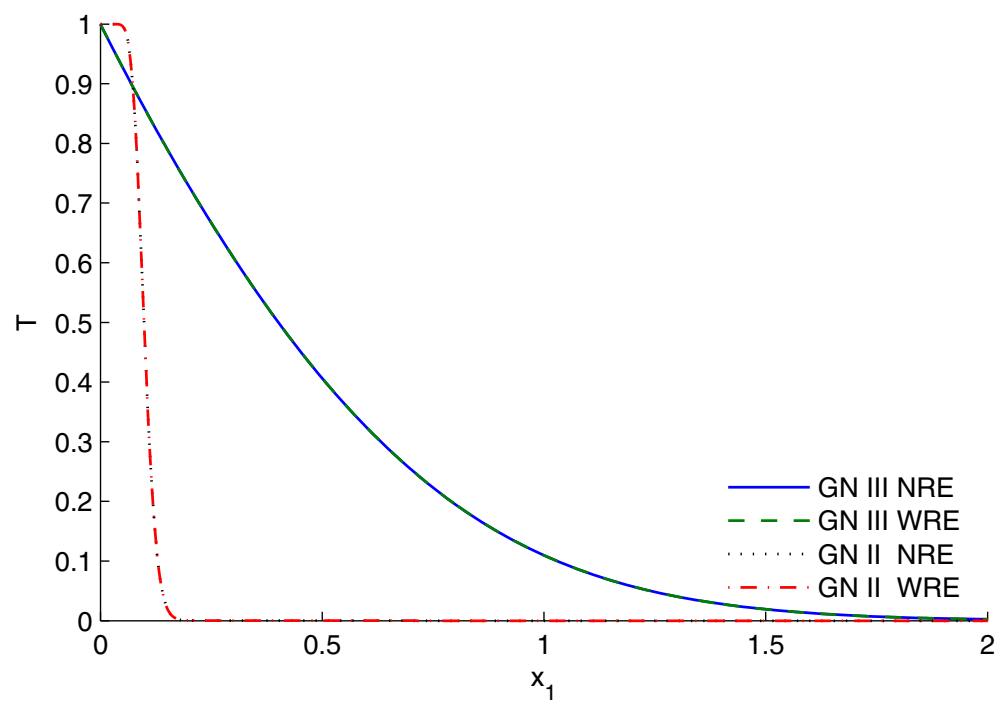

Figure 7. The temperature distribution at $t=0.2$ and $x_{2}=1$. 


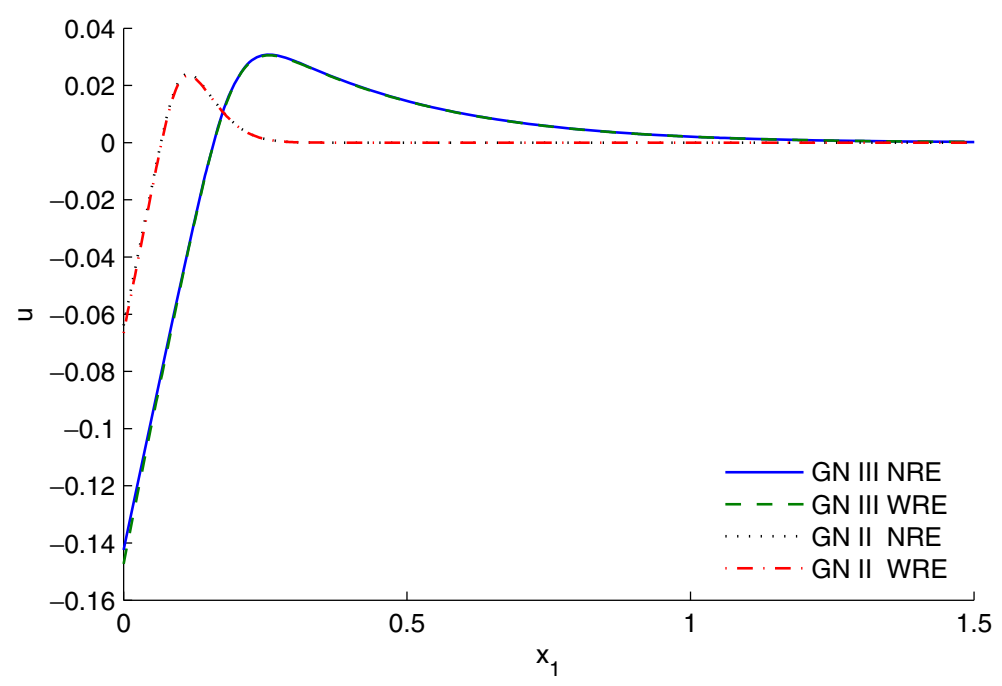

Figure 8. Horizontal displacement distribution $u$ at $t=0.2$ and $x_{2}=1$.

equation is hyperbolic. Among others, the introduction of a new internal variable, i.e., the thermal displacements with $\left(\varepsilon_{3}=0\right)$ leads to the theory without energy dissipation.

It can be found from figures 1-12 that, the physical quantities depend not only on time and space, but also depend on the characteristic parameter of the Green-Naghdi theory. Figures 1-6 are drawn to give comparison of the results obtained for displacements, temperature and stresses for two models of Green and Naghdi, namely GN model II and GN model III, against the thickness $x_{1}$ for different values of $t$ at $x_{2}=1$. Figure 1 shows the variation of temperature with $x_{1}$ and it indicates that temperature field has maximum value at the boundary and then decreases to zero. Figure 2 shows the variation of horizontal displacement with respect to $x_{1}$ at two different

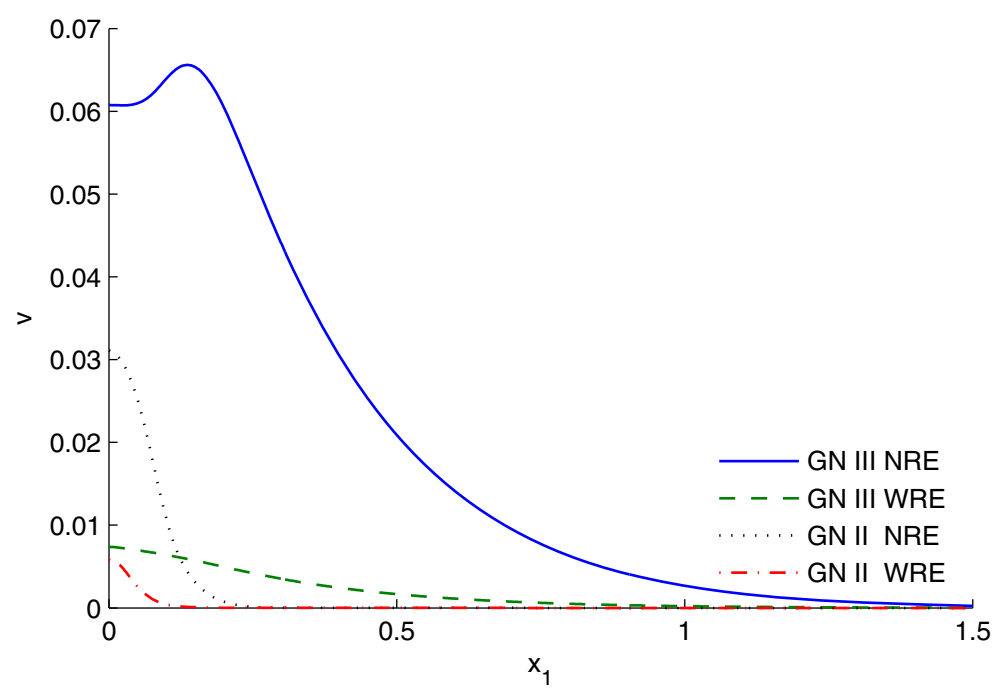

Figure 9. Vertical displacement distribution $v$ at $t=0.2$ and $x_{2}=1$. 


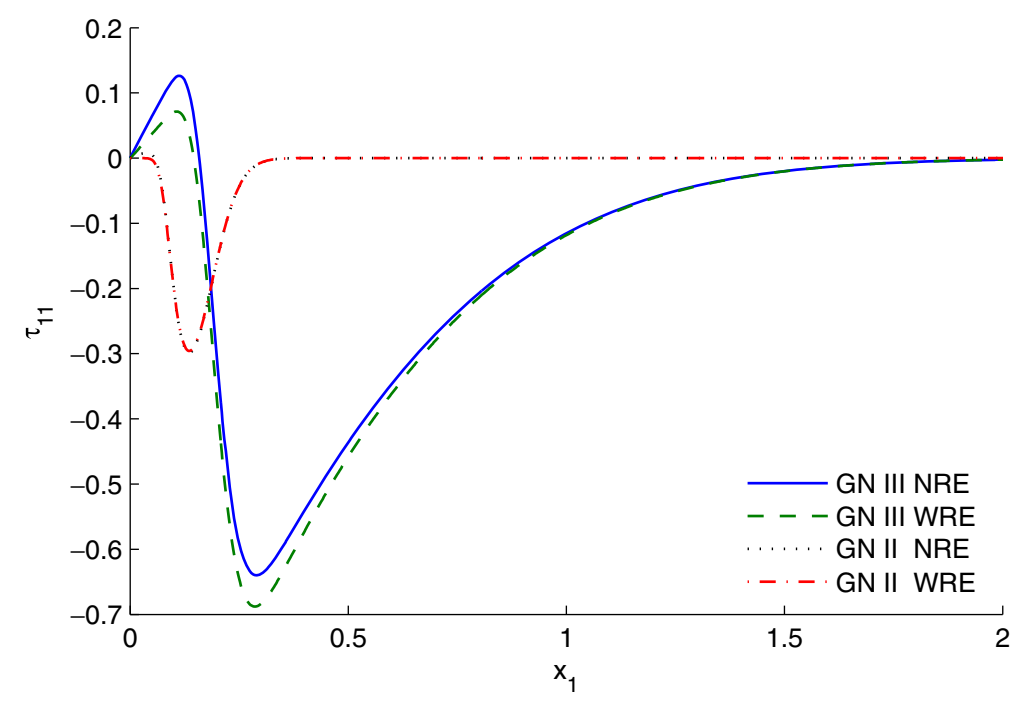

Figure 10. The distribution of stress component $\tau_{11}$ at $t=0.2$ and $x_{2}=1$.

times and it indicates that when the surface of the half-space is taken to be traction free, and the thermal shock applied on the surface, the displacement at $t=0.1$ shows a negative value at the boundary of the half space and it attains a stationary maximum value after some distance. Finally it decreases to zero value. The similar nature of variation is observed at $t=0.2$. Figure 3 exhibits the space variation of vertical displacement for different values of time in which we observed that, significant difference in the value of displacement is noticed for the different value of $t$ for the two types. We can also see that the maximum point of the displacement increases for the type (GNIII). Figures 4-6 show the variations of stress field with respect to $x_{1}$ for different values of

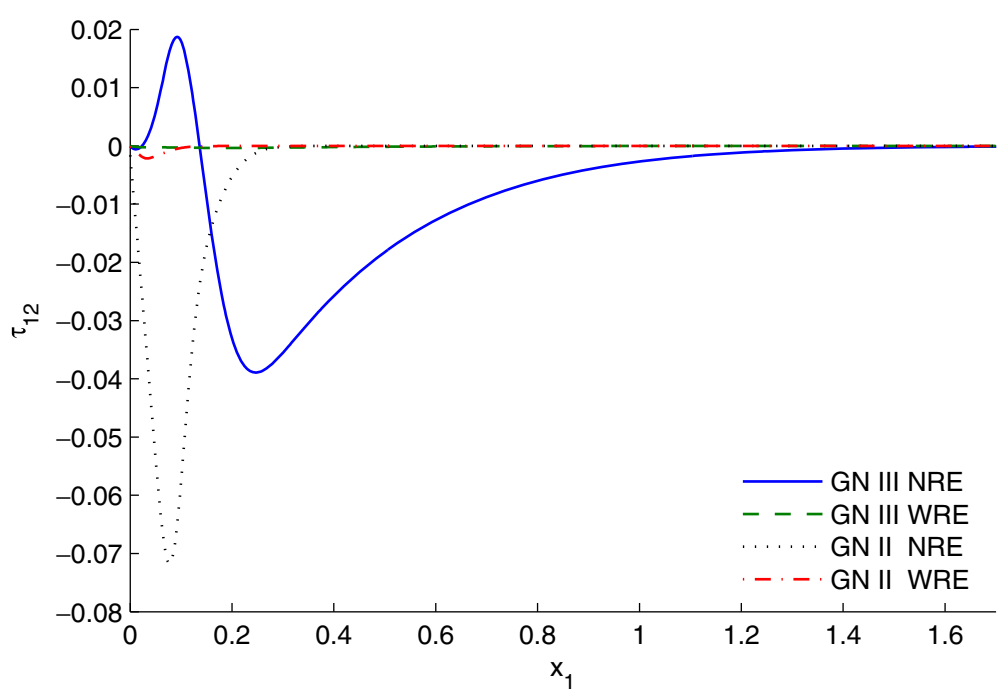

Figure 11. The distribution of stress component $\tau_{12}$ at $t=0.2$ and $x_{2}=1$. 


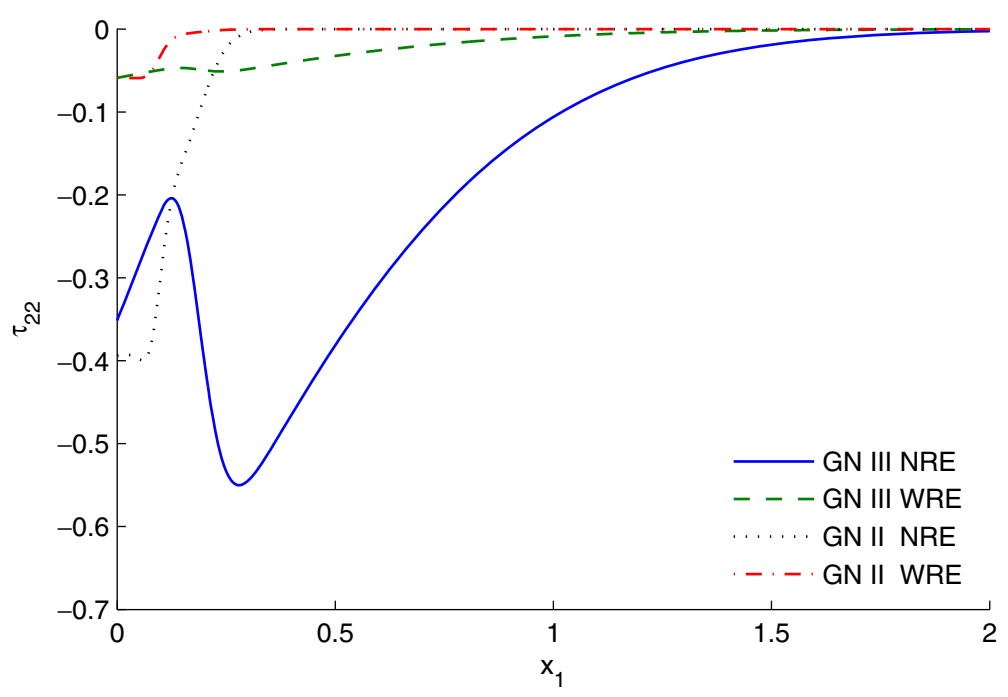

Figure 12. The distribution of stress component $\tau_{22}$ at $t=0.2$ and $x_{2}=1$.

time in which we observed that, the stresses $\tau_{11}$ and $\tau_{12}$ are zero at $x_{1}=0$ which satisfies the boundary conditions of the problem.

Figures 7-12 show the variation of the physical quantities with space $x_{1}$ at $t=0.2$ under two types with reinforcement (WRE) and without reinforcement (NRE i.e., $\alpha=0, \beta=0$ and $\mu_{L}-$ $\mu_{T}=0$ ). Also, the comparisons are made with the results predicted by these at $t=0.2$ for two models of Green and Naghdi, namely GN model II and GN model III. It is easy to see from figures 7 and 8 that the reinforcement has very small effect on the temperature and horizontal displacement while, it has a significant effect on the other field quantities as expected $\left(x_{1}\right.$-axis is the fibre-direction). Figures 9-12 show the fibre-reinforced has a high strength.

\section{Conclusion}

In this paper, we have investigated the solution of two-dimensional problem for a fibre-reinforced anisotropic thermoelastic half-space based on Green-Naghdi theory by using the finite element method. The differences of the field quantities predicted by the GN theory of two types are remarkable in the presence and absence of reinforcement. When $k \rightarrow 0\left(\varepsilon_{3}=0\right)$, Eq. (26)c reduces to the heat conduction equation under GN (type II) theory. The reinforcement has a great effect on the distribution of field quantities. The results carried out in this paper can be used to design various fibre-reinforced anisotropic thermoelastic elements under thermal load to meet special engineering requirements.

\section{References}

Abbas I A 2007 Finite element analysis of the thermoelastic interactions in an unbounded body with a cavity, Forsch Ingenieurwes 71: 215-222

Abbas I A and Abd-alla A N 2008 Effects of thermal relaxations on thermoelastic interactions in an infinite orthotropic elastic medium with a cylindrical cavity, Arch. Appl. Mech. 78: 283-293 
Abbas I A and Othman M 2009 Effect of rotation on thermoelstic waves with Green-Naghdi theory in a homogeneous isotropic hollow cylinder, Int. J. Industrial Mathematics 1(2): 121-134

Belfield A J, Rogers T G and Spencer A J M 1983 Stress in elastic plates reinforced by fibres lying in concentric circles, J. Mech. Phys. Solids 1: 25-54

Cook R D, Malkus D S and Plesha M E 1989 Concepts and applications of finite element analysis, $3^{\text {rd }}$ ed. John Wiley, New York

Dhaliwal R S and Sherief H H 1980 Generalized thermoelasticity for anisotropic media, Q. Appl. Math. 33: $1-8$

Green A E and Lindsay K A 1972 Thermoelasticity, J. Elast. 2: 1-7

Green A E and Naghdi P M 1991 A re-examination of the basic postulates of thermoemechanics, Proc. R. Soc. Lond. A 432: 171-194

Green A E and Naghdi P M 1992 On undamped heat waves in an elastic solid, J. Thermal Stress. 15: 253-264

Green A E and Naghdi PM 1993 Thermoelasticity without energy dissipation, J. Elast. 31: 189-208

Hashin Z and Rosen W B 1964 The elastic moduli of fibre reinforced materials, J. Appl. Mech. 31: 223-232

Lord H and Shulman Y 1967 A Generalized Dynamical Theory of Thermoelasticity, J. Mech. Phys. Solid 15: 299-309

Reddy J N 1993 An introduction to the finite element method, $2^{\text {nd }}$ ed. McGraw-Hill, New York

Sengupta P R and Nath S 2001 Surface waves in fibre-reinforced anisotropic elastic media, Sãdhana 26: $363-370$

Singh S J 2001 Comments on Surface waves in fibre-reinforced anisotropic elastic media, by Sengupta and Nath Sãdhanã 26: 363-370. Sãdhanã 27: 1-3 (2002).

Singh B 2006 Wave propagation in in thermally conducting linear fibre-reinforced composite materials, Arch. Appl. Mech. 75: 513-520

Tian X, Shen Y, Chen C and He T 2006 A direct finite element method study of generalized thermoelastic problems, Int. J. Solids Struct. 43: 2050-2063

Youssef H and Abbas I A 2007 Thermal shock problem of generalized thermo-elasticity for an infinitely long annular cylinder with variable thermal conductivity, Computational Methods in Science and Technol. 13(2): 95-100

Zienkiewicz O C and Taylor R L 2000. The finite element method. Fluid dynamics, 5th ed. ButterworthHeinemann 